\title{
Simple wave interaction of an elastic string
}

\author{
L. J.F. Broer and E.W. C. van Groesen \\ With 1 figure and 1 table
}

(Received October 27. 1976)

\section{Introduction}

In this paper we shall consider some aspects of the dynamical behaviour of an elastic string. As is well known, the equations that describe such a string admit as special solutions simple waves running in one direction. We shall present some results concerning the behaviour of the string when two simple transverse waves running in opposite directions collide. The behaviour of an ideally elastic string after collision is investigated for small amplitude interacting waves.

The motion of the string is assumed to take place in a plane, and one endpoint is fixed at the origin 0 of an $x-y$ coordinate system. As an independent variable to characterize a material point of the string a material (= Lagrangian) coordinate is taken, say $m$, which measures the mass of the string from 0 to the point under consideration. The position of a point of the string can then be written at any instant $t$ as

$\boldsymbol{r}=\boldsymbol{r}(m, t)$,

where $\boldsymbol{r}$ is the two-component vector $(x, y)$.

For an elastic string this choice of independent variable $m$ is the most natural one. For an inextensible string, the arclength $s$ would have been a more appropriate choice.

The specific length $l$ (stretch) is defined as the length of the string per unit mass:

$l=\frac{d s}{d m}$

and hence $l=1 / \rho$, where $\rho$ is the mass per unit length. With this definition we have the following relation

$l^{2}=\boldsymbol{r}_{m} \cdot \boldsymbol{r}_{m}$,

343 which follows at once from $\boldsymbol{r}_{m} \cdot \boldsymbol{r}_{m}=\boldsymbol{r}_{s} \cdot \boldsymbol{r}_{s}\left(\frac{d s}{d m}\right)^{2}$
and the fact that $\boldsymbol{r}_{s}$ is a unit vector.

Concerning the physics of the string, it is assumed that it is completely flexible, so that there are no bending forces at all. Furthermore, the elasticity of the string is described by a function $E$ which gives the deformation energy per unit mass. It is assumed that $E$ is a function of the specific length only: $E=E(l)$. The choice of this function is to some extend arbitrary; a physical requirement is that there exists a stress-free configuration in which the string has no energy. This means that there exists a certain value $l^{*}$ for which $E\left(l^{*}\right)=0$ and $\sigma\left(l^{*}\right)=0$, where $\sigma(l)$ is the tension of the string defined by

$\sigma(l)=\frac{d E}{d l}$.

It is naturally to require $\frac{d E}{d l}>0$ for $l>l^{*}$, and to deal only with configurations for which the tension is positive at any place, thus $l>l^{*}$.

A string is said to be ideally elastic if it is described by

$E(l)=\frac{1}{2} y\left(l-l^{*}\right)^{2}$

for $l>l^{*}$, where $y$ is a positive constant, Young's modulus.

For the following, the quantities $a$ and $c$ are defined by

$$
\begin{aligned}
& a(l):=\left\{\frac{1}{l} \frac{d E}{d l}\right\}^{1 / 2}, \\
& c(l):=\left\{\frac{d^{2} E}{d l^{2}}\right\}^{1 / 2},
\end{aligned}
$$

where it is assumed that $\frac{d^{2} E}{d l^{2}}$ is positive for
$l>l^{*}$. 
Another assumption is that $c>a\left(\right.$ for $\left.l>l^{*}\right)$, which in view of the relation

$c^{2}-a^{2}=l \frac{d}{d l} a^{2}$

means that we assume that $a$ will increase upon stretching the string. Note that for an ideally elastic string all these assumptions are fulfilled and that $c$ is a constant: $c=Y$.

The equations of motion can easily be obtained from Hamilton's principle. The Lagrangian of the system is simply the difference of the total kinetic energy and the potential energy

$L=\int_{0}^{M}\left\{\frac{1}{2} \boldsymbol{r}_{t}^{2}-E(l)\right\} d m$,

where $M$ is the total mass of the string.

Variation of the action functional

$A:=\int_{t_{1}}^{t_{2}} L d t$

gives as the equations of motion

$\boldsymbol{r}_{t t}=\left(a^{2}(l) \boldsymbol{r}_{m}\right)_{m}$,

where we have used [1] and [4].

Of course, appropriate boundary conditions can also be obtained from the variational principle, but we shall not bother about these here. [Although these results will not be used in the sequel, it may be noticed that the eq. [6] have several constants of the motion and corresponding local conservation laws, such as an energy and a Bernoulli-like conservation law; c. f. Broer (1).]

The eq. [6] may be written in a more convenient form by defining the vector $l=\boldsymbol{r}_{m}$ and a velocity vector $v=r_{t}$. Then the equations are seen to be in the form of a set of quasilinear hyperbolic equations

$\left.\begin{array}{rl}\boldsymbol{l}_{t} & =\boldsymbol{v}_{m}, \\ \boldsymbol{v}_{t} & =\left(a^{2}(l) l_{m}\right.\end{array}\right\}$.

As has already been remarked, these equations admit as special solutions simple waves. The characteristic equation for this set of equations shows that there are four characteristic velocities: $\pm a$ and $\pm c$, with $a$ and $c$ given by [4] and [5]. The corresponding simple waves will be investigated presently.

The derivation of the equations as given above has been taken from a paper of Broer (1). A different derivation was given by Parker and
Varley (2), who also studied some aspects of the interaction of simple waves. In this last respect, subsequent work of Parker (3) and (4) has also to be mentioned.

\section{Simple waves}

When studying special solutions such as simple waves, it is necessary to get rid of boundary conditions. Therefore, from now on, we assume the string to be of infinite length, with the material coordinate $m$ running from $-\infty$ to $+\infty$.

\subsection{Longitudinal motion}

Solutions which travel along the $c$-characteristics turn out to be well known from the theory of longitudinal motion of an elastic bar: they correspond to motions of the string for which $l$ and $v$ have constant directions, thus motions for which the string remains along the $x$-axis say. The governing equations are then seen to be

$\left.\begin{array}{rl}l_{t} & =v_{m}, \\ v_{t} & =c^{2}(l) \cdot l_{m}\end{array}\right\}$.

These equations are already dealt with in Courant and Friedrichs (5), and we simply note the following results.

Riemann invariants $\alpha$ and $\beta$ can be found

$\alpha=\frac{1}{2}(v+w), \beta=\frac{1}{2}(v-w)$,

where $w$ is defined for $l>l^{*}$ by

$w(l):=\int_{l^{*}}^{l} c(\zeta) d \zeta$,

for which

$\frac{d \alpha}{d t}=0 \quad$ along $\frac{d m}{d t}=-C(\alpha-\beta)$,

$\frac{d \beta}{d t}=0 \quad$ along $\frac{d m}{d t}=C(\alpha-\beta)$,

wherein

$C(w):=c(l(w))$.

A simple longitudinal wave running to the right (to the left) corresponds to a solution with $\alpha \equiv 0(\beta \equiv 0$ respectively $)$.

Note that, except for an ideally elastic string for which $c$ is a constant, the characteristics depend on the solution. Consequently, initial 
wave profiles will distort: the solution may become multivalued unless shocks are introduced.

\subsection{Simple transverse waves}

In this paper the more important simple waves are those which are propagated along the $\pm a$ characteristics. They can be found by simply investigating for which solutions $\partial_{t}=-a \partial_{m}$ (for a solution running to the right). Substituting this requirement in the equations leads to

$$
\begin{aligned}
& -a \boldsymbol{l}_{m}=\boldsymbol{v}_{m}, \\
& -a \boldsymbol{v}_{m}=\left(a^{2}(l) l\right)_{m} .
\end{aligned}
$$

From this it follows that $a^{2}$ must be a constant for these solutions, which means that the specific length must be a constant. Hence, these simple waves are solutions at constant length (and thus constant tension). Because of the absence of longitudinal motion, they will be called simple transverse waves.

From these observations it follows that every simple transverse wave running to the right can be described as

$l=l_{0}\left(\begin{array}{c}\cos \theta^{\rightarrow} \\ \sin \theta^{\rightarrow}\end{array}\right)$

where $\theta^{\rightarrow}=\theta^{\rightarrow}\left(m-a_{0} t\right)$ is an arbitrary function of its argument and $a_{0}=a\left(l_{0}\right)$, and a possible corresponding velocity is given by

$v=a_{0} l_{0}\left(\begin{array}{r}1-\cos \theta^{\rightarrow} \\ \sin \theta^{-}\end{array}\right)$.

From this explicit representation of a simple transverse wave it follows at once that such a solution travels with constant velocity $a_{0}$ and is undeformed in shape: these simple waves are exceptional.

Let us now suppose that $\theta^{\rightarrow}$ has bounded support, thus

$\theta^{\rightarrow}(\zeta)=0$ for $\zeta \notin(-p, 0)$.

Then such a simple wave can be expressed in the original variable $\boldsymbol{r}$ by simply integrating $l$ with respect to $m$ :

$\vec{r}=\left(\begin{array}{l}l_{0} m \\ 0\end{array}\right)+l_{0} \int_{0}^{m-a_{0} t}\left(\begin{array}{l}\cos \theta^{\rightarrow}(\zeta)-1 \\ \sin \theta^{\rightarrow}(\zeta)\end{array}\right) d \zeta$.

Here integration constants have been chosen such that $\vec{r}=\left(\begin{array}{c}l_{0} m \\ 0\end{array}\right)$

to the right of the $a_{0}$ characteristic through the origin of the $(m, t)$ plane, i. e. for $m-a_{0} t>0$. In that case the simple wave is running into an undisturbed region: the prestrained string lying along the $x$-axis. Note that for $m-a_{0} t<-p$, $\boldsymbol{r}$ is independent of time and given by

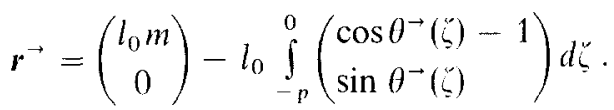

Furthermore, if $\int_{-b}^{0} \sin \theta^{\rightarrow}(\zeta) d \zeta=0, y=0$ for $m-a_{0} t<-p$, and the only notable effect after passage of the simple wave is a constant shift in $x$ for every $m$ (for which $m-a_{0} t<-p$ ).

In the same way simple transverse waves running to the left can be described: for instance, if $\theta^{\leftarrow}(\zeta)$ is an arbitrary function with bounded support

$\theta^{-}(\zeta)=0$ for $\zeta \notin$ for $(0, a)$,

a simple transverse wave running to the left into an undisturbed region, is given by

$\boldsymbol{r}=\left(\begin{array}{c}l_{0} m \\ 0\end{array}\right)+l_{0} \int_{0}^{m+a_{0}{ }^{2}}\left(\begin{array}{l}\cos \theta^{-}(\zeta)-1 \\ \sin \theta^{-}(\zeta)\end{array}\right) d \zeta$.

\subsection{Superposition of two simple transverse waves}

Consider now a string in which two of such transverse waves are present, one running to the right and the other to the left, both at the same constant specific length $l_{0}$.

If the separate simple transverse waves are described by [10], [11] and [12], [13] respectively, the combined result can be written as

$$
\begin{aligned}
\boldsymbol{r}= & \left(\frac{l_{0} m}{0}\right)+l_{0} \int_{0}^{m-a_{0} t}\left(\begin{array}{l}
\cos \theta^{\rightarrow}(\zeta)-1 \\
\sin \theta^{\rightarrow}(\zeta)
\end{array}\right) d \zeta \\
& +l_{0} \int_{0}^{m+a_{0} t}\left(\begin{array}{l}
\cos \theta^{\leftarrow}(\zeta)-1 \\
\sin \theta^{r-}(\zeta)
\end{array}\right) d \zeta .
\end{aligned}
$$

This expression indeed satisfies the equations of motion for negative values of $t$ : for $t<0$ the waves are running towards each other with constant speed $a_{0}$, separated by an undisturbed region where

$\boldsymbol{r}=\left(\begin{array}{c}l_{0} m \\ 0\end{array}\right)$ for $a_{0} t<m<-a_{0} t$, 
and at $t=0$ the two simple waves "collide". For positive $t$, thus after collision, expression [14] no longer satisfies the equations of motion. This is intuitively clear: due to the collision, the tension, which is a constant for negative $t$, will no longer remain constant. Hence some kind of longitudinal motion will be excited.

The exact solution for positive $t$ seems very difficult to obtain. Therefore, we shall approximate this solution in the next section under the assumption that the amplitudes of the initial simple transverse waves are small.

\section{Interaction of two infinitesimal simple transverse waves of an ideally elastic string}

\subsection{Small amplitude expansion}

By assuming the amplitudes of both the simple transverse waves before collision to be small, it is formally possible to expand the solution in terms of a small parameter. In that case we regard the prestrained string (with $l=l_{0}>l^{*}$ ) lying along the $x$-axis as an unperturbed configuration, and treat the initial simple transverse waves and the resulting motion after collision as perturbations.

Hence we put formally

$$
\left.\begin{array}{l}
x=l_{0}\left[m+\varepsilon \xi_{1}+\varepsilon^{2} \xi_{2}+\varepsilon^{3} \xi_{3}+\varepsilon^{4} \xi_{4}+\cdots\right] \\
y=l_{0}\left[\quad \varepsilon \eta_{1}+\varepsilon^{2} \eta_{2}+\varepsilon^{3} \eta_{3}+\varepsilon^{4} \eta_{4}+\cdots\right]
\end{array}\right\} .
$$

Substituting these expansions in the equations of motion [6] and equating the coefficients of each power of $\varepsilon$ to zero, an infinite series of equations for the expansion variables $\xi_{i}$ and $\eta_{i}$ result. However, it can be argued beforehand that in general the exact solution will not be approximated uniformly in time by a finite number of terms in [15]. Indeed, as we have seen above, for a non-ideally elastic string the longitudinal motion is such that shocks have to be introduced to prevent multivaluedness. In these cases it is known that an expansion as in [15] will not be valid uniformly in time.

To avoid these difficulties we shall from now on assume that the string is ideally elastic: then $c$, the propagation speed of the longitudinal motion is a constant and no multivaluedness or shocks will appear. With this restriction, substitution of [15] in [6] leads to the following set of equations for the expansion variables up to and including the fourth order:

$$
\begin{aligned}
& \square_{c} \xi_{1}=0 \text {, } \\
& \square_{a_{0}} \eta_{1}=0 \text {, } \\
& \mathrm{C}_{c} \xi_{2}=\left[\frac{1}{2} \eta_{1_{m}}^{2}\right]_{m} \text {, } \\
& \mathrm{Q}_{a_{0}} \eta_{2}=\left[\xi_{1_{m}} \cdot \eta_{1_{m}}\right]_{m} \text {, } \\
& \square_{c} \xi_{3}=\left[\eta_{1_{m}} \cdot \eta_{2_{m}}-\xi_{1_{m}} \cdot\left(\eta_{1_{m}}^{2}+\frac{1}{2} \check{\zeta}_{2_{m}}\right)\right]_{m}, \quad[20] \\
& \square_{a_{0}} \eta_{3}=\left[\xi_{1_{m}} \cdot \eta_{2_{m}}+\eta_{1_{m}} \cdot\left(\xi_{2_{m}}+\frac{1}{2} \eta_{1_{m}}^{2}-\xi_{1_{m}}^{2}\right)\right]_{m} \text {, } \\
& \square_{c} \xi_{4}=\left[\eta_{1_{m}} \cdot \eta_{3_{m}}-\frac{3}{8} \eta_{1_{m}}^{4}-\eta_{1_{m}}^{2} \cdot \check{\zeta}_{2_{m}}+\frac{1}{2} \eta_{2_{m}}^{2}\right. \\
& +\xi_{1_{m}} \cdot\left(-\frac{1}{2} \eta_{1_{m}} \cdot \eta_{2_{m}}+\frac{3}{2} \xi_{1_{m}} \cdot \eta_{1_{m}}^{2}\right. \\
& \left.\left.+\xi_{1_{m}} \cdot \xi_{2_{m}}\right)\right]_{m} \text {, } \\
& \square_{a_{0}} \eta_{4}=\left[\xi _ { 1 _ { m } } \cdot \left(\eta_{3_{m}}-\breve{\xi}_{1_{m}} \cdot \eta_{2_{m}}-\frac{5}{2} \xi_{2_{m}} \cdot \eta_{1_{m}}\right.\right. \\
& \left.-\frac{3}{2} \eta_{1_{m}}^{3}+\xi_{1_{m}}^{2} \cdot \eta_{1_{m}}\right) \\
& \left.+\xi_{3_{m}} \cdot \eta_{1_{m}}+\eta_{2_{m}} \cdot\left(\xi_{2_{m}}+\frac{3}{2} \eta_{1_{m}}^{2}\right)\right]_{m} \text {. }
\end{aligned}
$$

Here $\square_{c}$ and $\square_{a_{0}}$ denote the wave operators

$\square_{\mathrm{c}}=\left(c^{2}-a_{0}^{2}\right)^{-1} \cdot\left(\partial_{t}^{2}-c^{2} \partial_{m}^{2}\right)$,

$\square_{a_{0}}=\left(c^{2}-a_{0}^{2}\right)^{-1} \cdot\left(\partial_{t}^{2}-a_{0}^{2} \partial_{m}^{2}\right)$.

Note that the right hand side in each of these equations is a derivative with respect to $m$. This is a consequence of the fact that the original eq. [6] are in the form of a set of local conservation laws.

\subsection{Infinitesimal simple transverse waves}

Let us now first investigate how one simple transverse wave can be described in terms of the quantities $\xi$ and $\eta$. For such a wave running to the right we choose the vertical displacement to be

$y^{\vec{y}}=l_{0}\left[\varepsilon \eta_{\vec{l}}\right]$,

where $\eta_{1}=\eta_{1}\left(m-a_{0} t\right)$ is an arbitrary function of its argument. The results to be obtained can best be interpreted if it is assumed that $\eta_{\overrightarrow{1}}$ has bounded support, say

$\eta_{1}(\zeta)=0$ for $\zeta \notin(-p, 0)$.

Furthermore, it is assumed that $\eta_{1}$ is smooth, such that $\eta_{\overrightarrow{1}}=\eta_{1_{\xi}}=0$ for $\zeta=0$ and $\zeta=-p$. From the exact expression [9] for a simple transverse wave, the corresponding $\xi$ components can easily be found: as $\sin \theta^{\overrightarrow{ }}=\varepsilon \eta_{1_{m}}$, it follows that 


$$
\begin{aligned}
\overrightarrow{x_{m}} & =l_{0} \cos \theta^{\rightarrow}=l_{0}\left[1-\varepsilon^{2} \overrightarrow{\eta_{1_{m}}}\right]^{1 / 2} \\
& =l_{0}\left[1-\varepsilon^{2} \cdot \frac{1}{2}{\overrightarrow{1_{m}}}^{2}-\varepsilon^{4} \cdot \frac{1}{8} \eta_{1_{m}}^{4}+\cdots\right] .
\end{aligned}
$$

From this it follows that we may take

$\xi_{\overrightarrow{1}}=\xi_{\overrightarrow{3}}=\xi_{5}=\cdots=0$.

Furthermore, from

$\xi_{2_{m}}=-\frac{1}{2} \eta_{1_{m}}^{2}, \quad \xi_{4_{m}}=-\frac{1}{8} \eta_{1_{m}}^{-4}$,

$\xi_{2}$ and $\xi_{4}$ can be found by integration. If integration constants are chosen such that $\xi_{2}=\xi_{4}=0$ for $m-a_{0} t>0$, these functions are denoted by $\overrightarrow{\xi_{2}}$ and $\xi_{4}$.

In this way an infinitesimal simple transverse wave running to the right into an undisturbed region is described up to and including fourth order of $\varepsilon$.

In the same way an analogous wave running to the left into an undisturbed region can be described: if we choose

$y^{\leftarrow}=l_{0}\left[\varepsilon \eta_{1}^{\leftarrow}\right]$,

where

$\eta_{1}^{\leftarrow}=\eta_{1}^{\leftarrow}\left(m+a_{0} t\right)$

and

$\eta_{1}^{-}(\zeta)=0 \quad$ for $\quad \zeta \notin(0, p)$,

corresponding $\xi$ components can be found: $\xi_{1}^{-}=\xi_{3}^{-}=\xi_{5}^{-}=0$ and $\xi_{2}^{-}$and $\xi_{4}^{-}$such that $\xi_{4}^{-}=\xi_{4}^{--}=0$ for $m+a_{0} t<0$. Again, $\eta_{1}^{--}$is assumed to be smooth such that $\eta_{1_{\zeta}}=\eta_{1 ;}^{\leftarrow}=0$ for $\zeta=0$ and $\zeta=p$. [For convenience, especially for transparency of figure 1 , we have chosen the supporting intervals of $\eta_{1}$ and that of $\eta_{1}^{\leftarrow}$ of the same length.]

\subsection{Interaction of the two inf initesimal waves}

As follows from the end of section 2, the expressions

$x=l_{0}\left[m+\varepsilon^{2}\left(\xi_{2}+\xi_{2}^{\leftarrow}\right)+\varepsilon^{4}\left(\xi_{4}+\xi_{4}^{\leftarrow-}\right)\right]$,

$y=l_{0}\left[\varepsilon\left(\eta_{1}+\eta_{1}\right)\right]$

satisfy the equations of motion for negative $t$ (up to $0\left(\varepsilon^{5}\right)$ ), and describe the simple transverse waves, as defined above, running towards each other and colliding at $t=0$.

To describe the behaviours for $t>0$, we put

$\left.\begin{array}{ll}\xi_{i}=\xi_{i}+\xi_{i}^{-}+\xi_{i}^{\mathrm{int}} & i=1,2,3, \ldots \\ \eta_{1}=\eta_{1}+\eta_{1}^{-}+\eta_{1}^{\mathrm{int}} ; \eta_{i}=\eta_{i}^{\mathrm{int}} & i=2,3, \ldots\end{array}\right\}$, where the interaction terms satisfy

$\xi_{i}^{\mathrm{int}}=\eta_{i}^{\mathrm{int}} \equiv 0 \quad$ for $\quad t<0, \quad$ for $\quad i=1,2,3, \ldots$.

Substituting [28] in the eqs. [16]-[23], we arrive at a set of equations for the interaction terms. Some results may immediately be noticed:

First of all, $\eta_{1}^{\text {int }}$ and $\xi_{1}^{\text {int }}$ are identically zero as they satisfy the homogeneous wave equation with zero initial values [29].

As $\xi_{1} \equiv 0$, it follows in the same way that $\eta_{2}^{\text {int }}=0$. Likewise, from $\eta_{2}=\xi_{1} \equiv 0$ it follows that $\xi_{3}^{\text {int }}=0$, and then from $\eta_{2}=\xi_{3} \equiv 0$ it follows that $\eta_{4}^{\text {int }} \equiv 0$. Resuming we have

$\xi_{1}=\zeta_{3}=\eta_{2}=\eta_{4} \equiv 0$.

The first non zero interaction term is of second order in $\varepsilon: \xi_{2}^{\text {int }}$. The governing equation follows from [18], using the fact that this equation is satisfied for $\xi_{2}$ and $\eta_{1}$, and for $\xi_{2}^{<}$and $\eta_{1}^{-}$:

$\square_{c} \xi_{2}^{\text {int }}=\left[\overrightarrow{\eta_{1_{m}}} \cdot \eta_{1_{m}}^{\leftarrow}\right]_{m}$.

The source term of this equation has bounded support in the $(m, t)$-plane, viz. the area $O C^{\prime} A C$ in figure 1.

[In figure 1 a point $P^{\prime}$ corresponds to the point $P$ in the following way: if $P=\left(m_{p}, t_{p}\right)$,

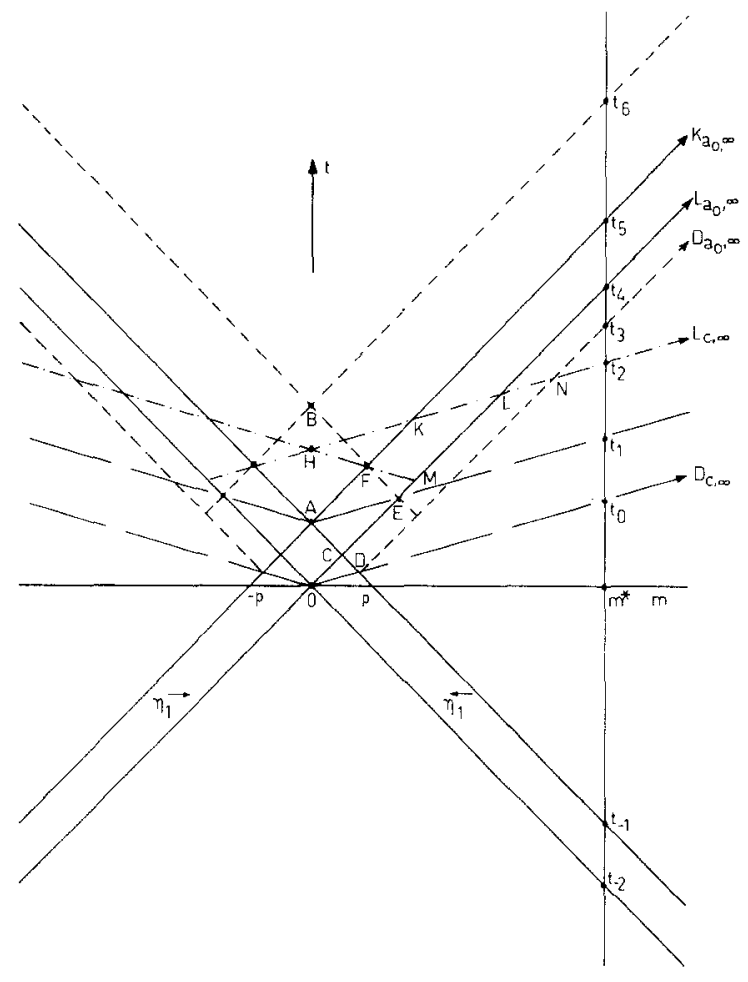

Fig. 1. See text 
then $P^{\prime}:=\left(-m_{p}, t_{p}\right)$. Note that the symmetry with respect to the $t$-axis in the figure is a consequence of the fact that the supporting intervals of $\eta_{1}$ and of $\eta_{1}^{<-}$are chosen to be of equal length. This choice simplifies the interpretation of the figure, but is of course not necessary.]

From elementary facts about the solutions of inhomogeneous wave equations, the following results are obtained from [31] supplied with zero initial values at $t=0$ (as follows from [29]):

Below the $\pm c$ characteristics through the point 0 in the $(m, t)$-plane, $\xi_{2}^{\text {int }}$ is identically zero. Furthermore,

$\xi_{2}^{\mathrm{int}}=$ constant on any $c$ characteristic issuing from $O C A$

$\xi_{2}^{\text {int }}=$ constant on any $-c$ characteristic issuing from $O C^{\prime} A$.

These last results imply that for $m>m_{C}, \xi_{2}^{\text {int }}$ behaves as a simple longitudinal wave running to the right: $\xi_{2}^{\text {int }}=\xi_{2}^{\text {int }}(m-c t)$ for $m>m_{C}$, and likewise $\xi_{2}^{\text {int }}=\xi_{2}^{\text {int }}(m+c t)$ for $m<m_{C^{\prime}}=$ $-m_{c}$. Above the $\pm c$ characteristics through $A$, $\xi_{2}^{\text {int }}$ is again identically zero:

$\xi_{2}^{\text {int }}=0 \quad$ for $\quad-c\left(t-t_{A}\right)<m<c\left(t-t_{A}\right), t>t_{A}$.

This follows from the observation that the domain of dependence of an arbitrary point $P$ in this area is actually the source area $O C^{\prime} A C O$ and, because of the fact the right hand side of [31] is a derivative with respect to $m$, the surface integral over the source area reduces to a line integral over the boundary:

$$
\begin{aligned}
& \xi_{2}^{\text {int }}(P)=\frac{c^{2}-a_{0}^{2}}{2 c} \iint_{O C^{\prime} A C O}\left[\overrightarrow{1_{1_{m}}} \cdot \eta_{1_{m}}^{\leftarrow}\right]_{m} d m d t \\
& =\frac{c^{2}-a_{0}^{2}}{2 c} \int_{0}^{t_{A}} \cdot\left\{\left.\eta_{1_{m}} \cdot \eta_{1_{m}}^{\leftarrow}\right|_{O C A}-\left.\eta_{1_{m}} \cdot \eta_{1_{m}}^{\leftarrow}\right|_{O C^{\prime} A}\right\} d t .
\end{aligned}
$$

Now, because of the assumed smoothness properties of $\eta_{\overrightarrow{1}}$ and $\eta_{1}^{\leftarrow}, \eta_{1_{m}}=0$ at $O C$ and $C^{\prime} A$, and $\eta_{1_{m}}^{\leftarrow}=0$ at $O C^{\prime}$ and $A C$. Hence the result [32]. Along the same lines it can be shown that

$\xi_{2_{m}}^{\mathrm{int}}=0$ at the $\pm c$ characteristics issuing from 0 and $A$.

The next order term to be considered is $\eta_{3}^{\text {int }}$. The governing equation follows from [21]

$$
\begin{aligned}
\square_{a_{0}} \eta_{3}^{\text {int }}= & {\left[\eta_{1_{m}}^{2} \cdot \eta_{1_{m}}^{\leftarrow}+\eta_{1_{m}} \cdot \eta_{1_{m}}^{\leftarrow-2}\right.} \\
& \left.+\xi_{2_{m}}^{\text {int }} \cdot\left(\eta_{1_{m}}+\eta_{1_{m}}^{\leftarrow}\right)\right]_{m},
\end{aligned}
$$

and this equation has to be supplied with homogeneous initial values at $t=0$. Again, the source term of this equation is non-zero only in a bounded domain of the $(m, t)$ plane, the area $O D C E A E^{\prime} C^{\prime} D^{\prime} O$ in figure 1. In this figure, $+a_{0}$ characteristics have been drawn issuing from the points $D$ and $E^{\prime}$ and $-a_{0}$ characteristics issuing from the points $D^{\prime}$ and $E$. Below the broken line $D_{a_{0}, \infty}^{\prime} D^{\prime} O D D_{a_{0}, \infty}, \eta_{3}^{\text {int }}$ is identically zero. Along the same lines as was done above for $\xi_{2}^{\text {int }}$ it can be shown, using result [33], that $\eta_{3}^{\text {int }}=0$ above the $a_{0}$-characteristics issuing from $B$, i.e.

$$
\begin{array}{r}
\eta_{3}^{\text {in }}=0 \text { for }-a_{0}\left(t-t_{B}\right)<m<a_{0}\left(t-t_{B}\right) \\
\text { for } t>t_{B} .
\end{array}
$$

Furthermore, it is not difficult to see that

$\eta_{3}^{\text {int }}=$ constant on any $a_{0}$ characteristic issuing from $B E$ or $C D$

and

$\eta_{3}^{\text {int }}=$ constant on any $-a_{0}$ characteristic issuing from $B^{\prime} E^{\prime}$ or $C^{\prime} D^{\prime}$.

This implies that for $m>m_{E} \eta_{3}^{\text {int }}$ behaves as a solution propagating undeformed in shape with constant velocity $a_{0}: \eta_{3}^{\text {int }}=\eta_{3}^{\text {int }}\left(m-a_{0} t\right)$ for $m>m_{E}$; likewise, $\quad \eta_{3}^{\text {int }}=\eta_{3}^{\text {int }}\left(m+a_{0} t\right)$ for $m<m_{E^{\prime}}=-m_{E}$.

The last interaction term that will be considered is $\xi_{4}^{\text {int }}$. Its equation follows from [22]:

$$
\begin{aligned}
& \square_{c} \xi_{4}^{\text {int }}=\left[-\frac{9}{4} \eta_{1_{m}}^{\overrightarrow{2}^{2}} \cdot \eta_{1_{m}}^{\leftarrow-2}-\frac{3}{2}\left(\eta_{1_{m}}^{\overrightarrow{3}} \cdot \eta_{1_{m}}^{-}+\eta_{1_{m}} \cdot \eta_{1_{m}}^{\leftarrow-3}\right)\right. \\
& \left.+\xi_{2_{m}}^{\text {int }} \cdot\left(\eta_{1_{m}}+\eta_{1_{m}}^{\leftarrow}\right)^{2}+\eta_{3_{m}}^{\text {int }}\left(\eta_{1_{m}}+\eta_{1_{m}}^{\leftarrow}\right)\right]_{m} \text {. }
\end{aligned}
$$

Due to the presence of the last term in the right hand side of [37], the source has no bounded support in the $(m, t)$-plane. Nevertheless, in large parts of the $(m, t)$-plane $\xi_{4}^{\text {int }}$ can be shown to be constant. This is seen by drawing the $+c$ characteristic through $F^{\prime}$ and the $-c$ characteristic through $F$ (see fig. 1). Then we have that $\xi_{4}^{\text {int }}=0$ below the $\pm c$ characteristic issuing from 0 , and

$\xi_{4}^{\mathrm{int}}=$ constant on any $+c$ characteristic issuing from $D C L$,

$\xi_{4}^{\text {int }}=$ constant on any $-c$ characteristic issuing from $D^{\prime} C^{\prime} L^{\prime}$. 
Furthermore,

$\xi_{4}^{\text {int }}=\xi_{4.00}^{\text {int }}=$ constant in the unbounded area

$$
L_{a_{0}, \infty} L L_{c, \infty} \text {, }
$$

i. e. in the area consisting of those points $(m, t)$ which lie above the $+c$ characteristic issuing form $L$ and below the $+a_{0}$ characteristic issuing form $L$. This follows at once from the observation that, because of the result [36], the value of $\xi_{4}^{\text {int }}$ at an arbitrary point of this area can be expressed as

$$
\begin{aligned}
-\frac{c^{2}-a_{0}^{2}}{2 c} & \left\{\left.\int_{t_{M}}^{t_{r}} \eta_{3_{m}}^{\mathrm{int}} \cdot \eta_{1_{m}}^{-}\right|_{M} F^{\prime} d t\right. \\
& \left.+\left.\int_{t_{k}}^{t_{L}} \eta_{3_{m}}^{\mathrm{int}} \cdot \eta_{1_{m}}\right|_{K L} d t\right\}
\end{aligned}
$$

In the same way it can be shown that

$\xi_{4}^{\text {int }}=$ constant in the unbounded area $L_{a_{0}, \infty}^{\prime} L^{\prime} L_{c, \infty}^{\prime}$.

Using the result [36] once again, one may deduce that

$\xi_{4}^{\mathrm{int}}=$ constant on any $+a_{0}$ characteristic issuing from $K L$,

$\xi_{4}^{\text {int }}=$ constant on any $-a_{0}$ characteristic issuing from $K L^{\prime}$.

With the final observation, again based on [36], that

$\xi_{4}^{\mathrm{int}}=\xi_{4,0}^{\mathrm{int}}=$ constant in the unbounded area

$$
K_{a_{0}, \infty}^{\prime} K^{\prime} H K K_{a_{0}, \infty},
$$

and that this constant is given by

$$
\begin{aligned}
\xi_{4.0}^{\text {int }}= & \frac{c^{2}-a_{0}^{2}}{2 c} \cdot \int_{t_{M}}^{t_{F}}\left\{-\left.\eta_{3_{m}}^{\mathrm{int}} \cdot \eta_{1_{m}}^{\leftarrow}\right|_{M^{\prime} F^{\prime}}\right. \\
& \left.+\left.\eta_{3}^{\mathrm{int}} \cdot \eta_{1_{m}}\right|_{M F}\right\} d t,
\end{aligned}
$$

the function $\xi_{4}^{\text {int }}$ is known everywhere.

The results obtained can best be interpreted by fixing a specific value of $m$ and considering the corresponding $x$ and $y$ values as function of $t$. For instance, if we take $m=m^{*}>m_{N}$, the two most important observations for $t>0$ are the passage of an excited longitudinal wave, partly of order $\varepsilon^{2}$ and partly of order $\varepsilon^{4}$, and the passage of the disturbed transverse wave, where the disturbance in the $y$-coordinate is of order $\varepsilon^{3}$ and exists on a larger interval of time than the original transverse wave should have done in the absence of interaction terms.
These results are summarized in more detail in table 1, for which the time intervals can be read of from figure 1 , and where $\xi_{0}$ and $\xi_{0}$ denote the constant shifts in the value of $\frac{x}{l_{0}}$ due to the passage of the simple transverse wave running to the right and to the left respectively $\left(\xi_{0}\right.$ and $\xi_{0}^{\leftarrow}$ are of order $\left.\varepsilon^{2}\right)$.

Table 1

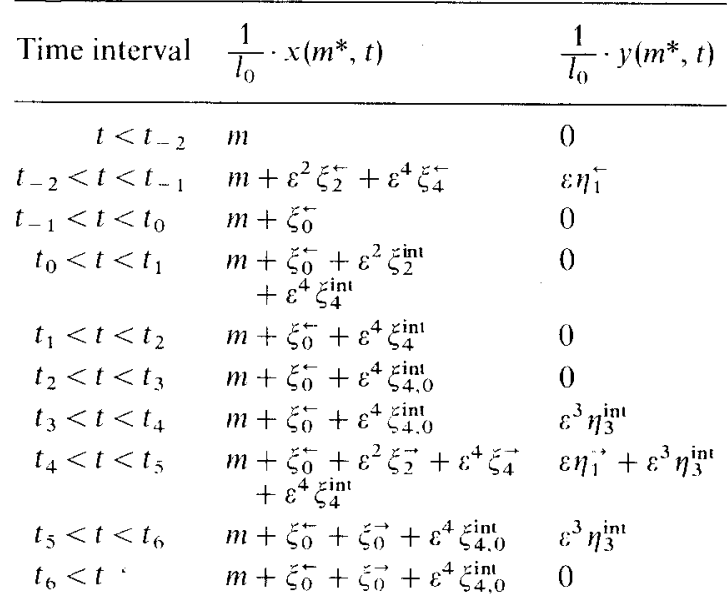

Note that from [32], [36], [38] and [39] it follows that the actual behaviour of the string due to the excited longitudinal wave and the disturbed transverse wave is independent of the point of observation provided $m>m_{N}$ : for another point $m_{1}>m_{N}$ the same behaviour of the string is found as for $m^{*}$, apart from a constant time shift and a difference in the length of the time interval $\left[t_{2}, t_{3}\right]$.

To conclude, we note that in case of completely symmetric initial simple transverse waves, i.e. if

$\eta_{1}(\zeta)=\eta_{1}^{\leftarrow}(-\zeta)$ for $\zeta \in(-p, 0)$,

the resulting solution is symmetric with respect to the $t$-axis. In that case, $\xi_{0}+\xi_{0}^{-}=0$ and $\xi_{4,0}=0$, and after passage of the disturbed transverse wave $\left(t>t_{6}\right.$ at $\left.m=m^{*}\right)$ the string is found in the same position as it was before the passage of the initial simple transverse wave $\left(t<t_{-2}\right.$ at $\left.m=m^{*}\right)$, up to and including fourth order terms in $\varepsilon$.

\section{Summary}

The equations for the two-dimensional motion of a completely flexible elastic string can be derived from a Lagrangian. The equations of motion possess four 
characteristic velocities, to which the following four simple wave solutions correspond: leftward and rightward propagating longitudinal and transverse waves. The latter are exceptional (constant shape). By expanding the solution about a steady solution the interaction of simple waves may be studied. A typical result is the following: As a consequence of their interaction two transverse waves running into opposite directions emit a longitudinal wave and undergo themselves a translation over a finite distance but remain otherwise unchanged. The results are also valuable for a full comprehension of the interaction process of simple waves on inextensible strings.

\section{Zusammenfassung}

Die Gleichungen für die ebene Bewegung eines ideal flexiblen elastischen Seils lassen sich aus einer LagrangeFunktion ableiten. Die Bewegungsgleichungen besitzen vier charakteristische Geschwindigkeiten. Ihnen entsprechen vier mögliche einfache Wellen: links- und rechtsläufige longitudinale und transversale Wellen. Die letzteren sind ausgeartet (von konstanter Form). Durch Entwicklung der Lösung um eine stationäre Lösung läßt sich die Wechselwirkung einfacher Wellen studieren. Ein typisches Ergebnis ist das folgende: Infolge ihrer Wechselwirkung emittieren zwei gegen. läufige transversale Wellen eine longitudinale Welle und erleiden selbst eine Versetzung um eine endliche Strecke, bleiben aber sonst ungeändert. Die Ergebnisse sind auch wertvoll für ein tieferes Verständnis der Wechselwirkung einfacher Wellen auf undehnbaren Seilen.

\section{References}

1) Broer, L. J. F., J. Eng. Math. 4, 195 (1970).

2) Parker, D. F., E. Varley, Quart. Journ. Mech. Appl. Math. 21, 329 (1968). (1970)

3) Parker, D. F., J. Mech. Phys. Solids 18. 331

4) Parker, D. F.. Int. J. Non-Linear Mech. 5, 589 (1970).

5) Courant, R., K. O. Friedrichs, Supersonic Flow and Shock Waves (New York 1948).

\section{Discussion}

Parker: Do I gather that this interaction region goes right off to infinity in the fourth order terms? Can this be interpreted as just a change in the transverse simple wave, that is a slightly different transverse wave? If you integrate the equations of the fourth order, is the solution still a simple wave?

can Groesen: It is still a simple wave, if you were far enough along in the $(m, t)$-plane: c.f. formula [39]

Broer: The longitudinal wave given off can be regarded as energy radiated due to the collision of the two transverse waves, and then the resulting transverse waves must be of slightly lower energy.

Bürger: You started off with two waves which are confined to finite regions of space. But when these interact they continually emit waves so that eventually there will be a disturbance filling the whole region.

can Groesen: That is quite right. If one goes on to calculate the higher order terms they will eventually fill the whole $(m, t)$ plane above the \pm ( characteristic issuing from 0 .

Buggisch: What happens in the situation when the speed of the longitudinal wave grows off to infinity. that is, when you are dealing with an inextensible string?

ian Groesen: In that case the boundary condition must be taken into account. When the two transverse waves collide, there is an instantaneous change in tension throughout the whole string. We have looked at this problem for certain boundary conditions and the equations for transverse waves after the interaction look much the same as they do in the extensible case.

Becker: Is it possible to choose the form of the two transverse waves in such a way, that there is no longitudinal wave generated?

can Groesen: No, it is not possible if these waves are running in opposite directions. This can be shown directly from the equations of motion, or by an investigation of the source of the interaction terms, $\xi_{2}^{\text {int }}$ for example, which gives rise to the longitudinal wave.

Broer: This question is equivalent to asking: Does there exist a solution in which the length of the string remains unchanged, so that no longitudinal wave is generated, and the answer is, the only solution is the single transverse simple wave.

Authors' address :

Prof. Dr. L. J.F. Broer

Ir. E. W. C. van Groesen

Department of Physics

Eindhoven University of Technology

Postbus 513

Eindhoven (The Netherlands) 